\title{
An optically isolated digital interface for the SKED system
}

\author{
MICHAEL G. GRISHAM and LLOYD J. FREI \\ University of Iowa, Iowa City, Ioua 52242
}

\begin{abstract}
A digital interface to support the SKED software system in recent versions of the PDP-8 computer. One printed circuit card of interface logic and optical isolators can be installed in the OMNIBUS to provide 24 input and 36 output lines to external panels containing input simulation switches and input or output indicators.
\end{abstract}

The increasing use of minicomputers for the control of experiments and data collection in psychology reflects both decreasing cost and the availability of process control languages that allow the experimenter to quickly translate procedures into computer programs. The cost of a minicomputer system to support a process control language depends primarily on the hardware interface the language requires. A recent survey of process control languages for psychology (Wood, Sette, \& Weiss, 1975) revealed that the SKED software system (Snapper, Stephens, \& Lee, 1974) involves the least expensive interface. Specifically, a Digital Equipment Corporation (DEC) PDP- ${ }^{1}$ running SKED is interrupted only by a single real-time clock; thus, the hardware needed to provide response interrupts and external counters or timers for ACT (BRS/LVE) and SCAT (Grason-Stadler) is unnecessary for SKED.

Despite the relative simplicity of SKED hardware, the potential user generally lacks the technical support to design and construct his own digital interface. The excellent inexpensive interface available from State Systems $^{2}$ should be considered. The State Systems BUS-ALL hardware (Butler, 1974; Cobez \& Snapper, 1976 ) requires ground to a $28-\mathrm{V}$ relay as input and provides relay contact closures as output. A PDP-8/L equipped with a 36-input and 36-output BUS-ALL has worked in support of undergraduate research projects in our department for 1 year, with no hardware problems except a single failure of an integrated circuit chip in its clock. Connection of the BUS-ALL to a PDP-8/L requires no skills more esoteric than inserting cards into a connector block, installing cables linking the connector block to the computer and to external panels, and attaching power, inputs, and outputs to terminal strips.

The State Systems BUS-ALL is nearly immune to either misuse or electrical noise, since the relays at input and output electrically isolate the interface and

Design of this equipment was supported by Biomedical Sciences Support Grant FR-07035 from the National Institutes of Health. Funds were awarded by the Office of the Vice President for Educational Development and Research, University of Iowa. Reprints are available from M. G. Grisham, Department of Psychology, University of Iowa, lowa City, Iowa 52242. computer from the external world. The PDP-8/L, however, is not a current-generation machine. The BUS-ALL interface must be linked to the newer PDP-8/E, F, M, and PDP-8/A computers by an external I/O bus (KA8-E). The KA8-E plugs into the OMNIBUS inside newer PDP-8s and makes the newer machines electrically and logically equivalent to their predecessors in terms of interfacing. In addition to the BUS-ALL, other peripherals (e.g., magnetic tape) designed for the PDP-8/L and often available used at low cost may be connected to newer machines equipped with the KA8-E. Despite this potential advantage of KA8-E installation, two unfortunate consequences also follow. First, computer voltages are carried outside the computer cabinet and may be subject to noise or loss. Second, and relevant only for very demanding programs approaching the worst case, inputs are processed and outputs provided at approximately one-third the speed available from newer machines.

Another alternative for the PDP-8/E, F, M, and A computers is the standard DEC digital interface for OMNIBUS use. A DR8-E digital I/O card, providing 12 input and 12 output lines, is installed directly in the OMNIBUS and connected by cables to external supporting circuitry consisting of switch filters on inputs and relay drivers on outputs. Computer voltages are carried outside the computer cabinet and isolation of the computer from external equipment is not accomplished. The external circuitry is generally constructed from DEC printed circuit modules that must be interconnected by user wire-wrapping or a connector block. This wiring task involves substantial design and construction time, particularly when compared to the completely prewired BUS-ALL connector block and relay panels. However, the major drawback to the DEC digital interface is cost. The DEC system provides input and output at $\$ 26$ per line, not including external isolation, indicators, and input simulation switches. A portion of the higher cost of the DEC DR8-E provides features which SKED does not need, such as response interrupt handling and special commands which selectively clear input and output buffers. Indeed, the SKED run-time program must be modified to handle the DEC DR8-E. SKED software 
uses only two digital interface commands: transfer the input buffer to the accumulator and transfer the accumulator to the output buffer.

Our goal in designing the present hardware was an optimal SKED interface for the PDP-8/E, F, M, and Aan interface that needs minimal external supporting circuitry, keeps computer voltages inside the cabinet, isolates external equipment from the computer, and is easily installed. The basic component is a printed circuit which plugs directly into the OMNIBUS and provides 24 input and 36 output lines, in comparison to the 12 input and 12 output lines available from the DR8-E. However, this increased capability actually requires fewer integrated circuit chips per board, since DR8-E commands which SKED does not use have been omitted. Two or more cards may be used in a single machine if OMNIBUS slots are available. Optical isolators on the card itself protect the computer from misuse of input and output lines. Ribbon cables carry signals from the isolators to external input and output panels. In 6 months of 24-h daily use, the only hardware failures in two prototype systems were two output isolators.

An optical isolator contains a light-emitting diode (LED) and a Darlington light-sensitive transistor. The input lines from the external input panel provide current to illuminate the LED. Light from the LED produces collector current flow in the Darlington and the resulting higher voltage at the emitter junction of the Darlington is available as an input on the interface card. On the output lines, the LED is illuminated by circuitry on the interface card and the Darlington output is available to drivers on the external output panel. The driver will switch any voltage from 1 to $30 \mathrm{~V}$ dc at $300 \mathrm{~mA}$ maximum current, sufficient to operate most solid state devices, relays, or miniature lamps directly. Specifics regarding 6-, $12-$, and $24-\mathrm{V}$ dc external power supplies are presented subsequently in the theory of operation. External relays are required if high current or ac devices are to be controlled. Since information is transferred in and out of the computer by light, excessive voltage or current applied to the interface destroys only the isolator or output driver involved. Both isolators and drivers are mounted in sockets.

The input circuitry outside the computer cabinet includes a simple resistor-capacitor arrangement to eliminate contact bounce artifacts, switches to simulate input occurrence, and LEDs to indicate the status of each input. A LED indicator is also associated with each output driver. Like the optical isolator, the high-intensity LED is better and cheaper than the conventional component it displaces; the more usual incandescent lamp must be mounted in an expensive socket so that it can be frequently replaced. Approximate total cost of the present circuit card itself, components, and external panels is $\$ 10$ per line, assuming the user puts the interface together. Alternatively; the completely wired interface will soon be available from State Systems.

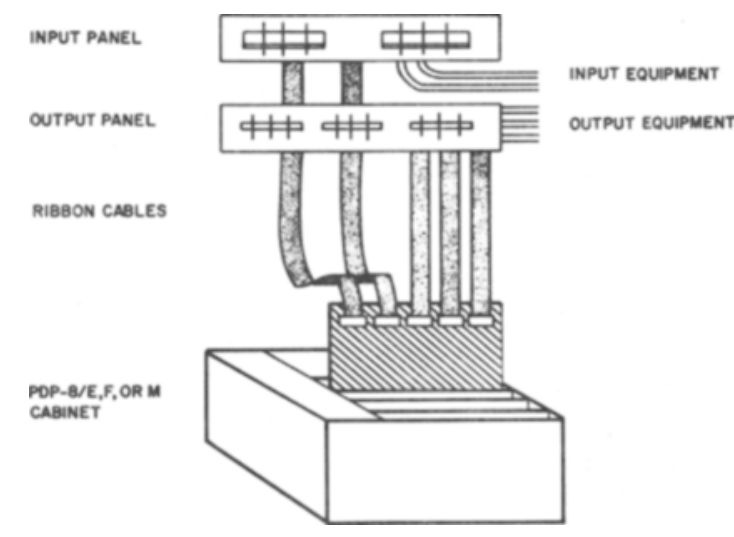

Figure 1. Interface card partially inserted into computer cabinet with two connecting cables to the input panel and three to the output panel.

As shown in Figure 1, installation of the interface involves inserting the card into any unused OMNIBUS slot in a PDP-8/E, F, M, or PDP-8/A. A device code is assigned by setting three small switches mounted on the card to form an octal digit. Any code between 10 and 17 can be selected. The input panel (switches and LED indicators) and the output panel (LED indicators only) must be mounted near the computer and connected to the card by five ribbon cables. Finally, external equipment is wired to the terminal strips on the input and output panels. Any current run-time program available from the SKED Users Group ${ }^{3}$ will work immediately in the PDP-8/A with the present interface and the 8/A $100-\mathrm{Hz}$ clock that DEC supplies. The SKED Users Group can provide a minor run-time program revision that permits SKED to use a DEC DK8-EC real-time clock in the PDP-8/E, F, or $M$, while the present digital interface works immediately.

The following brief theory of operation is presented to allow technical evaluation of the interface. $\mathrm{I} / \mathrm{O}$ decoding will not be described, since a standard programmed I/O (Digital Equipment Corporation, 1973) design is used. Figure 2 shows the component layout.

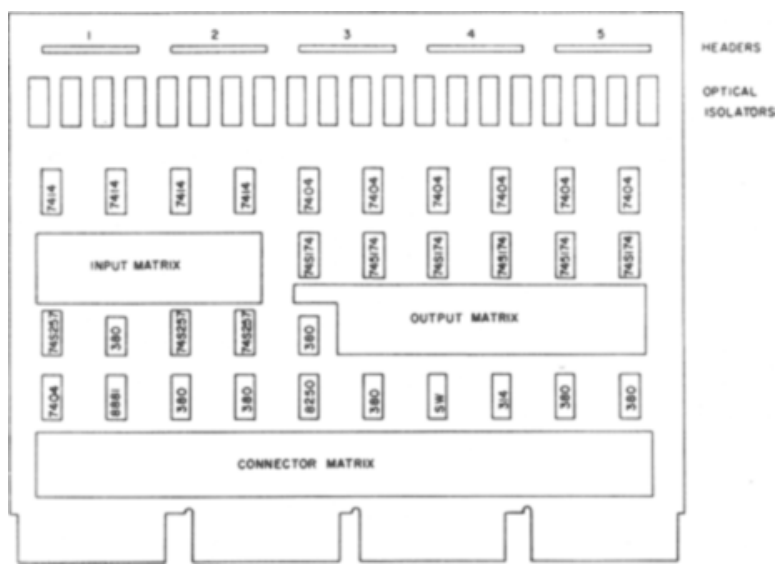

Figure 2. Component layout on the interface card. 


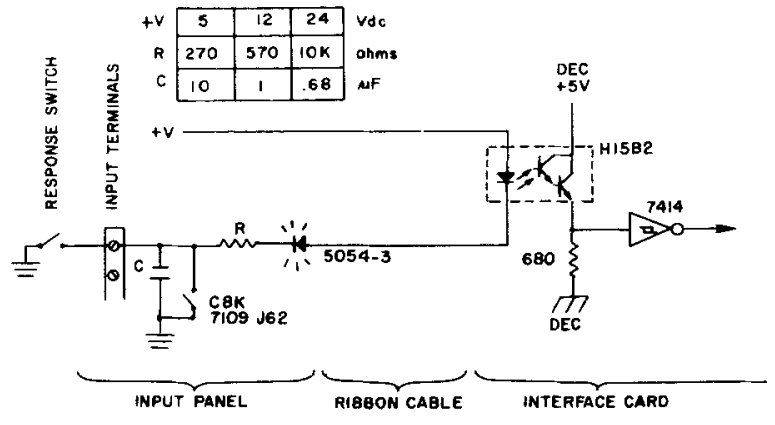

Figure 3. Schematic of one input line through the input panel and a portion of the interface card.

The card is mechanically identical to a single DEC quad board. Printed circuit appears on both sides of the card and plated-through holes carry signals from one side to the other as necessary. Plating through also eliminates the reed for soldering connections on the component side. The OMNIBUS edge connectors at the bottom of the card are gold-plated. Each of the five 14-pin AMP 1-872334 headers at the top of the board carries 12 signal lines and external power to a ribbon cable terminated with an AMP 2-87175-9 connector. The 14th pin is used to mechanically key each header to a particular ribbon cable connector. GE H15B2 optical isolators are mounted three per integrated circuit socket directly below the headers.

Headers 1 and 2 are for input cables. Figure 3 presents a schematic of the input panel circuitry and a portion of the interface card circuitry for one input line from a switch closure response. Collection of response duration requires a double-throw response switch and two input lines. The anode side of each input isolator LED is supplied positive voltage from the external power supply. The cathode side of each input isolator LED is wired through the input panel indicator LED to a current-limiting resistor. The values of the input panel resistor $(R)$ and capacitor $(C)$ are determined by the particular external voltage $(+V)$ and by the resistance-capacitance time constant needed to integrate switch contact bounce. The input isolator Darlington drives a 7414 Schmitt trigger inverter that shapes the input signal. Headers 1 and 2 provide $A$ and $B$ inputs, respectively, to three $74 \$ 257$ two-line to one-line data selector/multiplexers. A $61 \times 4$ decoded by an 8250 on the OMNIBUS line selects $A$ inputs and a $61 \times 5$ selects $B$ inputs. Either $61 \times 4$ or $61 \times 5$ causes $\mathrm{C} 0$ and $\mathrm{C} 1$ to go low, jam transferring current input levels to the accumulator. The $\mathrm{x}$ represents the switch-determined second octal digit of the interface card device code.

Headers 3, 4, and 5 are for output cables. The output schematic is shown in Figure 4. Data from the OMNIBUS is gated to a register bus line that presents the same binary word to all three output registers. The particular

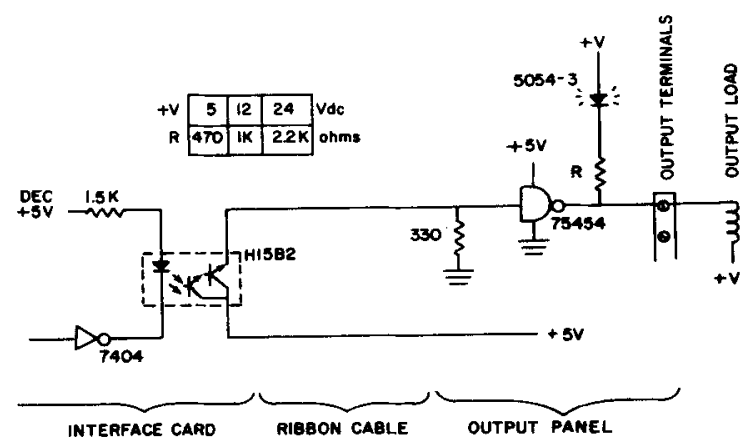

Figure 4. Schematic of one output line from a portion of the interface card through the output panel.

register loaded is determined by outputs from the 8250 decoder which are anded with a buffered TP3 pulse. Each register consists of two 74S174 flip-flops. IOTs $61 \times 1,61 \times 2$, and $61 \times 3$ jam transfer accumulator contents to headers 3,4 , and 5 , respectively. When a particular accumulator bit is set, a one output from the appropriate flip-flop allows a 7404 inverter to sink current from the corresponding output isolator LED. The isolator Darlington then provides voltage to a 75454 driver on the output panel. The value of the current-limiting resistor (R) for the output indicator LED depends on the external dc voltage (+V). A 7805 regulator on the output panel supplies $+5 \mathrm{~V}$ to the isolator and driver if $+\mathrm{V}$ is over $6.7 \mathrm{~V}$. The regulator is bypassed when $+\mathrm{V}$ is $5 \mathrm{~V}$.

Minicomputers should make it simpler to undertake or modify many experimental procedures. However, as Sidowski (1976) has noted, a remarkable discrepancy exists between the number of psychologists who own minicomputers and the number who report using them. SKED is a versatile and quickly learned process control and data collection language applicable to a wide range of paradigms. Completely packaged SKED software and hardware is now available for any version of the most common minicomputer, the DEC PDP-8. Even ignoring the possibility of a unique contribution from research on real-time response contingencies (Uttal, 1972), many psychology laboratories could benefit from easier and more flexible control of experiments.

\section{REFERENCES}

Butler, F. E. The BUS-ALL: An inexpensive set of interface for the SKED System. Behavior Research Methods \& Instrumentation, 1974, 6, 171-173.

Conez, R. I., \& SNAPPER, A. G. Interfacing for the SKED System. Behavior Research Methods \& Instrumentation. 1976, 8, 73-75.

Digital EQUIPMENT CoRPORATION. Small computer handbook. Maynard, Mass: Author, 1973.

Sidowski, J. B. On-line instrumentation in psychology: Dildo or the real thing? Behavior Research Methods \& Instrumentation, 1976, 8, 52-56. 
Snapper, A. G., Stephens, K. R., \& Lee, D. M. The SKED software system. Kalamazoo: SKED Users Group, 1974.

UTtal, W. R. Misuse, abuse, overuse, and unuse of on-line computer facilities by psychologists. Behavior Research Methods \& Instrumentation, 1972, 4, 55-60.

Wood, R. W., Sette, W. F., \& WeIss, B. Interfacing the experimenter to the computer: Languages for psychologists. American Psychologist, 1975, 30, 230-238.

\section{NOTES}

1. PDP is a registered trademark of Digital Equipment Corporation, Maynard, Massachusetts.

2. State Systems, Inc., P. O. Box 2215, Kalamazoo, Michigan 49003.

3. SKED Users Group, Department of Psychology, Western Michigan University, Kalamazoo, Michigan 49008. 